\title{
CRISPR/Cas9-mediated knockout of APOC3 stabilizes plasma lipids and inhibits atherosclerosis in rabbits
}

\author{
Yiwen Zha ${ }^{1 \dagger}$, Yaoyao Lu ${ }^{1 \dagger}$, Ting Zhang ${ }^{2}$, Kunning Yan ${ }^{1}$, Wenwen Zhuang ${ }^{1}$, Jingyan Liang ${ }^{3,45^{*}}$ (D), \\ Yong Cheng ${ }^{2,5^{*}}$ and Yingge Wang ${ }^{3,4,6^{*}}$
}

\begin{abstract}
Background: High levels of apolipoprotein C3 (APOC3) can lead to hypertriglyceridemia, which increases the risk of cardiovascular disease. We aim to create APOC3-knockout $(K O)$ rabbits and explore the effects of APOC3 deletion on the occurrence and development of atherosclerosis.

Methods: An sgRNA anchored to exon 2 of APOC3 was designed to edit embryo genomes using the CRISPR/Cas9 system. The founder rabbits were sequenced, and their lipid profile, inflammatory cytokines, and atherosclerotic plaques were analyzed.

Results: When given a normal chow (NC) diet, all APOC3-KO rabbits had 50\% lower triglyceride (TG) levels than those of the matched age control group. Additionally, their plasma lipoprotein lipase increased. When fed a highfat diet, APOC3 deficiency was observed to be more conducive to the maintenance of plasma TG, total cholesterol, and low-density lipoprotein cholesterol levels, and the inhibition of the inflammatory response and the protection against atherosclerosis in rabbits.
\end{abstract}

Conclusion: APOC3 deficiency can delay the formation of atherosclerosis-induced HFD in rabbits, indicating this is a novel therapeutic target to treat atherosclerosis.

Keywords: Apolipoprotein, Triglycerides, Atherosclerosis, CRISPR/Cas9, Rabbit

\section{Introduction}

APOC3 is a key regulator of plasma triglycerides (TG) and shows significant correlation with plasma very lowdensity lipoprotein (VLDL) levels [1, 2]. Hypertriglyceridemia represents an independent risk factor for cardio- and cerebrovascular diseases [3, 4]. Although in vivo studies on APOC3 have been mainly based on

\footnotetext{
* Correspondence: jyliang@yzu.edu.cn; chengyong@yzu.edu.cn; yinggewang279@hotmail.com

†Yiwen Zha and Yaoyao Lu contributed equally to this work.

${ }^{3}$ Institute of Translational Medicine, Medical College, Yangzhou University, Yangzhou 225001, Jiangsu, China

${ }^{2}$ College of Veterinary Medicine, Yangzhou University, Yangzhou 225009, Jiangsu, China

Full list of author information is available at the end of the article
}

mouse models, immense differences in lipid metabolism have been shown to exist between mice and humans. Carriers with APOC3 mutations had a $40 \%$ lower risk of coronary heart disease [5], but no protective effect of APOC3 deficiency on atherogenesis was observed in knockout (KO) mice [6]. Mice secrete VLDL containing either apolipoprotein B100 (Apob100) or apolipoprotein B48 (Apob48), while human and rabbit VLDL only contains APOB100. As opposed to mice, in which highdensity lipoprotein (HDL) is the main plasma lipoprotein, rabbits and humans have LDL-rich lipoprotein profiles [7]. All animal models of human disease have strengths and limitations, and domestic rabbits

\section{BMC}

(c) The Author(s). 2021 Open Access This article is licensed under a Creative Commons Attribution 4.0 International License, which permits use, sharing, adaptation, distribution and reproduction in any medium or format, as long as you give appropriate credit to the original author(s) and the source, provide a link to the Creative Commons licence, and indicate if changes were made. The images or other third party material in this article are included in the article's Creative Commons licence, unless indicated otherwise in a credit line to the material. If material is not included in the article's Creative Commons licence and your intended use is not permitted by statutory regulation or exceeds the permitted use, you will need to obtain permission directly from the copyright holder. To view a copy of this licence, visit http://creativecommons.org/licenses/by/4.0/ The Creative Commons Public Domain Dedication waiver (http://creativecommons.org/publicdomain/zero/1.0/) applies to the data made available in this article, unless otherwise stated in a credit line to the data. 
(Oryctolagus cuniculus) continue to play significant roles in the study of lipid metabolism and histopathology [8].

CRISPR/Cas9 is machinery for editing genomes that forms a natural part of the bacterial defense mechanism and has been developed as a tool for genetic manipulation in mice [9], rabbits [10, 11], dogs [12], hamsters [13], etc. In comparison with earlier genome-editing technologies, e.g., zinc finger nucleases (ZFNs) and transcription activator-like effector nucleases [14], CRISPR/ Cas9 has superior targeting efficiency, is less expensive, and is easier to design and implement [15]. In addition, CRISPR-mediated genome editing is emerging as a therapeutic strategy for combating cardiovascular diseases [16]. We designed our exon 2-anchored CRISPR/ Cas9 system to generate APOC3-KO rabbits with biallelic mutations to study the relationship between APOC3 deficiency, abnormal lipid metabolism, and the formation and development of atherosclerosis in this study.

\section{Material and methods Animals}

New Zealand White rabbits were sourced from Yangzhou University's Animal Genetic Engineering Laboratory. The animals were kept in a barrier facility with a 12-h light-dark cycle at $24{ }^{\circ} \mathrm{C}$ and $55 \%$ humidity, and they were provided ad libitum access to water and fed twice a day with normal chow (NC) diet or high-fat diet (HFD) (TP 2R301, Trophic, Ltd., Nantong, China). Yangzhou University's Animal Care Committee approved all animal studies in this work.

\section{Cas9 mRNA and sgRNA preparation}

To design mutant loci of the rabbit APOC3 gene, we obtained sequences from NCBI (http://www.ncbi.nlm.nih. gov/) and designed CRISPR/Cas9 single-guide (sg) RNAs using the tool provided on the website: http://crispr.mit. edu. Then, we chose one sgRNA targeting the loci of rabbit APOC3 by anchoring to exon 2, as shown in Fig. 1.

\section{Zygote injection and embryo transplantation}

Female rabbits older than 6 months and fertile male rabbits were used to obtain embryos. Donor rabbits were superovulated with $60 \mathrm{IU}$ pregnant mare serum gonadotropin, and $72 \mathrm{~h}$ later, the donors and recipients were simultaneously administered $10 \mathrm{IU}$ human chorionic gonadotropin intravenously, and the donors were mated with male rabbits. To collect embryos for microinjection, they were aspirated $18-20 \mathrm{~h}$ after mating using PBS. Under a Leica inverted light microscope, the embryo cytoplasms were microinjected with a mix containing Cas9 mRNA (33 ng/ $\mu \mathrm{l})$ and sgRNA $(40 \mathrm{ng} / \mu \mathrm{l})$ in solution. The processed embryos were placed in M2 cushion fluid and incubated at $38^{\circ} \mathrm{C}, 5 \% \mathrm{CO} 2$ for $30 \mathrm{~min}$, and the oviducts of pseudo-pregnant female rabbits received $10-15$ of the resulting zygotes.

\section{Sequencing analysis of founder rabbits}

Tail biopsy specimens were taken for the extraction of genomic DNA using the phenol-chloroform method. PCR amplification of sgRNA target sites utilized the primers F: 5'-GCTCACCCAGCTGAGATCCAT-3' and R: 5' -CAAAGTGCTTACGGGCAGAGG-3'. After purifying with agarose gel electrophoresis, the PCR products were cloned into a pMDTM 19-T vector (Takara Bio, Inc., Japan), and Lasergene software (DNASTAR, Inc., USA) was employed for the sequencing and analysis of positive clones.

\section{Extraction and analysis of mRNA in liver and small intestine}

TRIzol reagent (Gibco; Thermo Fisher Scientific, Inc.) was used to extract the total liver and small intestine RNA, which was reverse-transcribed with the iScript cDNA Synthesis Kit (Bio-Rad, CA, USA). In qPCR, the Mini Opticon Real-Time PCR detection system (BioRad) was used with GoTaq qPCR Master Mix (Promega, WI, USA) to amplify specific cDNA. The relative mRNA quantity was calculated using the $2-\Delta \Delta \mathrm{Cq}$ method and

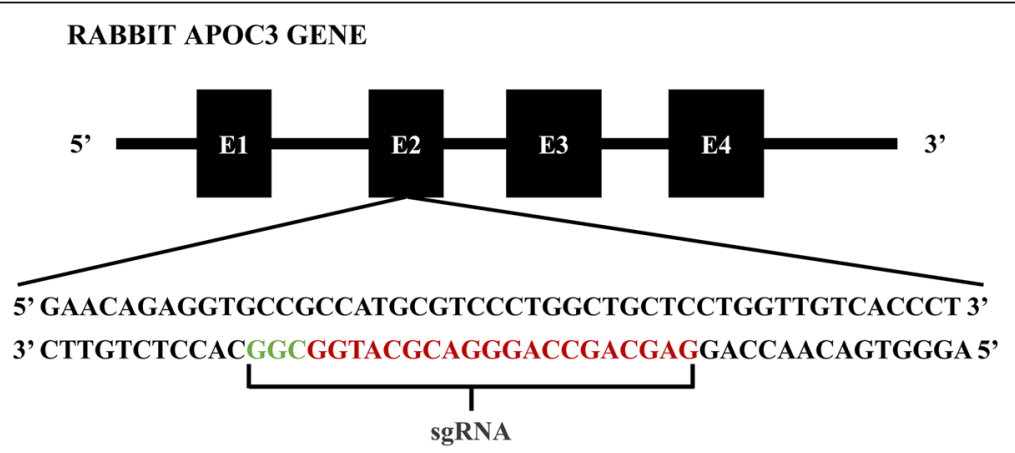

Fig. 1 Schematic illustration of CRISPR/Cas9-targeting sites of rabbit APOC3. An sgRNA was designed for anchoring to APOC3 exon 2. The sgRNAtargeting sequence is indicated in red, and the protospacer adjacent motif (PAM) sequence is indicated in green 
normalized to GAPDH. We completed triplicate qPCR reactions on three separate samples.

The sequences of the primers were as follows: APOC3: For 5' '-CCTCCCTTCTCAGCGTCATG-3', Rev. 5'GTCCCAGAACCCAGAGAACT-3' and GADPH: For 5'-CATGTTTGTGATGGGCGTGAACAA-3', Rev. 3'TAAGCAGTTGGTGGTGCAGGAT-3'.

\section{Off-target assay}

The CRISPR/Cas9 system is used to efficiently alter genes in living cells and organisms allowing any subsequent changes to the phenotype to be studied. However, certain off-target effects of this technology should be considered. The APOC3-KO rabbits were checked for off-target mutations by first predicting potential offtarget sites (POTS) in the sgRNAs with the CRISPR Design Tool (http://crispr.mit.edu/), then selecting the top five POTS for each sgRNA, which were PCR amplified with the primers shown in Table 1 and Sanger sequenced.

\section{Phenotypic examinations Analysis of lipids and apolipoproteins}

Samples of EDTA plasma were taken after $12 \mathrm{~h}$ of fasting. The plasma total cholesterol (TC), triglyceride (TG), LDL-cholesterol (LDL-C), and HDL-cholesterol (HDLC) levels were measured enzymatically with commercially available kits (Wako Pure Chemical Industries, Osaka, Japan). Western blotting was used to detect plasma APOC3, APOA1, APOE, and PCSK9; the following primary antibodies were used: goat anti-APOC3 (Genecreate, Wuhan, China), sheep anti-apoA1 (Bio-Rad AbD Serotec, Kidlington, UK), goat anti-APOE (Rockland Inc., Limerick, PA, USA), and mouse anti-PCSK9 (Abcam, Cambridge, MA, USA) polyclonal antibodies. Transferrin (Abcam, Cambridge, UK) was included as a loading control. The secondary antibodies were horseradish-peroxidase-conjugated donkey anti-goat IgG (Jackson Immuno Research Laboratories, West Grove, PA, USA), donkey anti-sheep IgG (Chemicon, Temecula,

Table 1 Primers for detection of possible off-target sites

\begin{tabular}{ll}
\hline Primers & off-targets of GCAGGGCCAGAGCCCAGGTG AGG \\
\hline off-targets-1 & 5' TCGTCGGCAGCGTCACAAAGTGCTTACGGGCAGA 3' \\
& 5' GTCTCGTGGCTCGGCTCCACTTCCTCCCTGCAG 3' \\
off-targets-2 & 5' TCGTCGGCAGCGTCGAGAAGAAGCACCACCCCTC 3' \\
& 5' GTCTCGTGGCTCGGACAGGGATCAAGGAAGGACTG 3' \\
off-targets-3 & 5' TCGTCGGCAGCGTCGGAAATGGTGAGTGAGCCCA 3' \\
& 5' GTCTCGTGGCTCGGCAGTCACACGGCTTAGTCGT 3' \\
off-targets-4 & 5' TCGTCGGCAGCGTCTCGTGAAGGACTCTCCACCA 3' \\
& 5' GTCTCGTGGCTCGGATCTCACCTTCGACAGCCG 3' \\
off-targets-5 & 5' TCGTCGGCAGCGTCCGACAGGGGTGGGGAAAC 3' \\
& 5' GTCTCGTGGCTCGGTTCCCAGAAACCTGCCCTC 3'
\end{tabular}

CA, USA), and goat anti-mouse IgG (Sangon Biotech, Shanghai, China).

\section{Analysis of plasma lipoprotein profiles}

Plasma lipoprotein fractions were determined by agarose gel electrophoresis. Plasma $(2 \mu \mathrm{l})$ samples were run on $1 \%$ agarose gel (Helena Laboratories, Saitama, Japan) electrophoresis and the neutral lipids stained with Fat Red 7B.

\section{Analysis of plasma HL, LPL}

The activities of hepaticlipase (HL) and LPL were assayed using commercial kits (Solarbio, Beijing, China).

\section{Analysis of plasma inflammation}

Plasma levels of interleukin (IL)-1 $\beta$ and tumor necrosis factor alpha (TNF- $\alpha)$ were ascertained using anti-rabbit ELISA (IL-1 $\beta$, Elabscience, Ltd., Wuhan, China; TNF- $\alpha$, Cusabio, Wuhan, China). Complete blood counts were measured using a BC-2800Vet auto hematology analyzer (Mindray Medical International Ltd., Shenzhen, China).

\section{Analysis of fat tolerance}

The rabbits were fasted for $12 \mathrm{~h}$, weighed, and orally gavaged with olive oil (Aladdin) at $10 \mathrm{ml}$.

per $\mathrm{kg}$ body weight using a $20 \mathrm{ml}$ syringe. Blood was collected before and after $1,3,5$, and $7 \mathrm{~h}$ of.

gavage, and the plasma TG levels at each time point were measured as already described. Oral fat tolerance tests (OFTT) were evaluated in terms of the accumulation and clearance of TG.

\section{Analysis of atherosclerosis and liver pathological changes} We analyzed the atherosclerotic lesions of the aorta using a previously published protocol [17]. In brief, the rabbits were sacrificed by lethal sodium pentobarbital injection. Their aortic trees were isolated and longitudinally opened and, after 24-h formalin fixation, stained using Sudan IV (Solarbio Life Science). Areas that stained positive were quantified using Image $J$ and expressed as the percentage of the total tissue area.

In subsequent histological analysis, the aortic root samples were paraffin-sectioned, treated with hematoxylin-eosin (HE) and Masson's trichrome (MT) stains, and incubated with monoclonal antibodies specific for macrophages $(M \phi)$ (clone: RAM11, Dako, CA, US) or the $\alpha$-smooth muscle actin in smooth muscle cells (clone: HHF35, Dako).

In accordance with the method described in a published report, we sectioned the formalin-fixed hearts into six blocks and embedded these in paraffin [18]. The left coronary artery was dissected to reveal cross-sections ( $5 \mu \mathrm{m} / \mathrm{slice})$ and stained with HE. After formalin fixing the fresh liver tissues for $48 \mathrm{~h}$, they were routinely 
embedded in paraffin, cut into $5-\mu \mathrm{m}$ serial sections, and processed for HE staining. To observe lipid accumulation, frozen sections of liver were stained with Oil-red O.

\section{Statistical analysis}

The data were expressed as mean \pm SD and compared by Student's t test. All statistical analyses were conducted on GraphPad Prism, and $P<0.05$ was used as statistical significance threshold.

\section{Results}

\section{Production of APOC $3 \mathrm{KO}$ rabbits and genotype assay}

We designed one sgRNA targeting the APOC3 anchored to exon 2 to generate mutant rabbits. Following in vitro transcription from the sgRNAa, the mRNAs and Cas9 mRNAs were co-injected into the rabbit zygotes. As shown in Tables 2, 144 embryos were injected with Cas9 mRNA, 101 of which were transferred to five pseudopregnant recipient rabbits (16-20 embryos per recipient). Three recipient rabbits became pregnant, and five pups were born (numbered AC1-AC5), two of which died on the day of birth (AC4 and AC5). All of the rabbits born (five in all) were sequenced. The three surviving founders were used for further phenotypic testing.

A pair of primers was designed to amplify the target fragment of APOC3 (F:5' -GCTCACCCAGCTGAGATC CAT-3', R:5'-CAAAGTGCTTACGGGCAGAGG-3'), which was sequenced to determine whether the gene was mutated; then the PCR products were sequenced by TA-clone to further determine the mutation type and efficiency. The PCR results in Fig. 2A revealed overlapping peaks for all five rabbits, suggesting that there may have been mutations, such as deletions, insertions, or frameshifts. The PCR products were ligated into the PMDTM 19- $\mathrm{T}$ vector, and the genomic DNA sequences are shown in Fig. 2B. The theoretical amino acid sequences of the APOC3 mutant alleles are provided in Fig. 2C. $\mathrm{AC} 1 \hat{\gamma}, \mathrm{AC} 2$ 웅, and $\mathrm{AC} 3$ ㅇ had frameshift mutations that ultimately led to the termination of translation. The

Table 2 Summary of Apoc3-KO rabbits generation and gene targeting efficiency

\begin{tabular}{llll}
\hline Number of microinjection & NO.1 & NO.2 & NO.3 \\
\hline No. of injected embryos & 51 & 56 & 37 \\
No. of transferred embryos & 43 & 38 & 20 \\
No. of recipients & 2 & 2 & 1 \\
No. of pregnancies & 2 & 1 & 0 \\
No. of births in total & 3 & 2 & 0 \\
No. of live pups & 2 & 1 & 0 \\
No. of mutants & 4 & 1 & 0 \\
Rate of mutations (\%) & 100 & & \\
\hline
\end{tabular}

amino acid profiles of $\mathrm{AC} 1(1)$ and $\mathrm{AC} 3(1)$ were identical, as were the amino acids of $\mathrm{AC} 1(2)$ and $\mathrm{AC2}(1)$.

Western blotting analysis showed that APOC3 was undetectable in the serum of the APOC3-KO rabbits (Fig. 2D). Furthermore, on measuring the RNA levels by RTqPCR, APOC3 was still detectable in the WT rabbit liver and small intestine, but expression was not detected in the founder rabbits (Fig. 2E). Finally, a total of five POTS were successfully PCR-amplified and Sanger sequenced, which did not reveal any overlapping peaks around the POTS. We concluded that genetic KO of APOC3 was achieved in the rabbit model by CRISPR/Cas9 gene editing technology.

\section{Analysis of lipids, apolipoproteins, and lipoproteins}

Evidence shows that APOC3 can effectively increase the level of circulating TG [19-21]. When the APOC3-KO rabbits were 2 months old, fasting blood samples were collected for lipid concentration detection. As predicted, the KO rabbits showed significantly lower plasma TG levels and higher plasma HDL-C levels than control rabbits of the same age under an NC diet (Table 3). However, there were no significant differences in plasma TC or LDL-C levels between the two groups.

To understand the effects of APOC3 on lipid metabolism in more detail, APOC3-KO and control rabbits were put on an HFD supplemented with $3 \%$ cholesterol and $10 \%$ soybean oil for 12 weeks at 6 months of age, and their fasting plasma lipid profiles were measured in the 6th and 12th weeks. The plasma of the wildtype (WT) rabbits appeared cloudy due to the high lipid content following high-fat feeding, whereas in the sera of the APOC3 $\mathrm{KO}$ rabbits, no obvious chylomicron (CM) aggregation was observed (Fig. 3A). According to the results, the levels of plasma TG, TC, and LDL-C increased significantly, while the levels of plasma HDL-C decreased significantly in all rabbits on an HFD; however, changes in the blood lipid profile of the control group were more obvious than those of the $\mathrm{KO}$ group (Table 3). After further analysis, we determined that the levels of TG and LDL-C in the control group plasma increased 13-18 and 17 times, while those in the KO group increased 6-10 and 12 times, respectively. The body weight statistics for the rabbits are shown in Supplementary Fig. 1. Due to the insufficient sample size, it was difficult to judge whether there was a difference in body weight between $\mathrm{KO}$ rabbits and WT rabbits after high fat feeding.

We further analyzed the rabbit plasma lipoprotein profiles by agarose gel electrophoresis after 6 weeks of highfat feeding, and this showed that fewer lipoproteins in the plasma of rabbits AC1-AC3 migrated from their location of origin to the pre- $\beta$-area, and more lipoproteins migrated to the $\alpha$-area than in the control rabbits (Fig. 


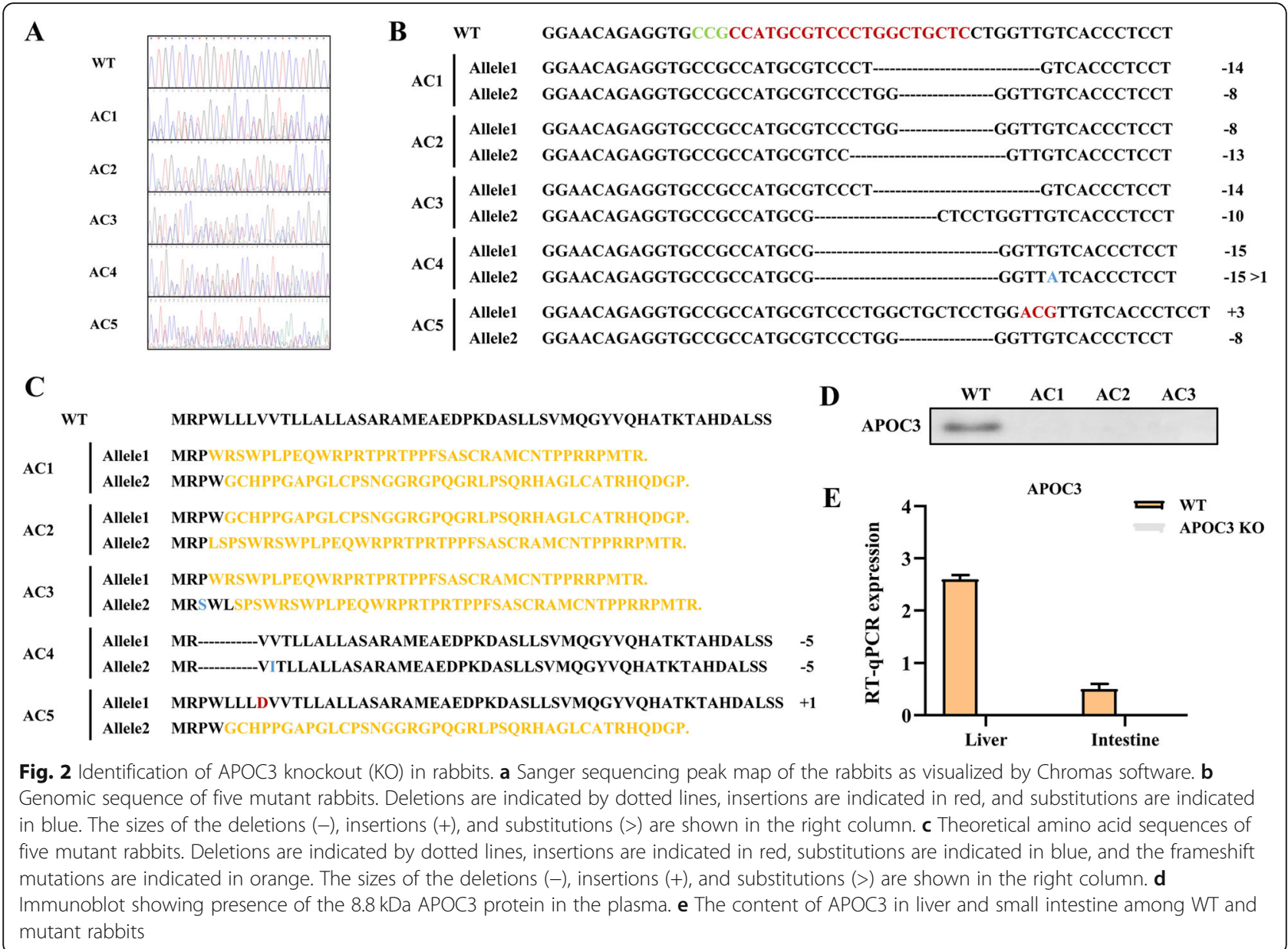

3A). Consistent with this, western blotting analysis showed significant differences in the plasma lipoprotein profiles of the WT and APOC3-KO rabbits; APOB and APOE were significantly reduced in the APOC3-KO rabbits, while APOA1 was enriched and led to the high HDL levels (Fig. 4B).
According to the results of OFTT test, the plasma TG level in the APOC3-KO rabbits was lower than that of the control group after olive oil gavage (Fig. 3C). Furthermore, after 12 weeks of HFD, plasma LPL activity was significantly increased in $\mathrm{KO}$ rabbits compared with control rabbits (Fig. 3D).

Table 3 Plasma levels of TC, TG, LDL-C, and HDL-C of the rabbits

\begin{tabular}{|c|c|c|c|c|c|}
\hline Diet & Group & TC (mg/dl) & TG (mg/dl) & LDL-C (mg/dl) & HDL-C (mg/dl) \\
\hline \multirow[t]{4}{*}{$\overline{N C}$} & $\mathrm{WT}(n=5)$ & $48.0 \pm 8.1$ & $59.9 \pm 13.2$ & $23.7 \pm 2.9$ & $28.7 \pm 4.4$ \\
\hline & $\mathrm{ACl} \hat{\circ}$ & $44.6 \pm 7.5$ & $23.8 \pm 11.8$ & $18.4 \pm 2.7$ & $31.1 \pm 5.1$ \\
\hline & $\mathrm{AC} 2$ 우 & $30.8 \pm 6.9$ & $36.3 \pm 8.7$ & $18.6 \pm 3.1$ & $21.5 \pm 6.3$ \\
\hline & $\mathrm{AC} 3$ ㅇ & $54.3 \pm 8.8$ & $30.5 \pm 6.5$ & $20.3 \pm 2.6$ & $33.7 \pm 3.8$ \\
\hline \multirow[t]{4}{*}{ HF (6W) } & $\mathrm{WT}(n=5)$ & $786.6 \pm 48.5$ & $877.4 \pm 69.8$ & $329.9 \pm 23.3$ & $26.4 \pm 9.6$ \\
\hline & $\mathrm{ACl} \hat{\mathrm{O}}$ & 288.5 & 256.0 & 125.3 & 43.8 \\
\hline & $\mathrm{AC} 2$ + & 318.8 & 285.7 & 159.3 & 45.1 \\
\hline & $\mathrm{AC} 3$ 우 & 355.3 & 298.5 & 201.2 & 39.2 \\
\hline \multirow[t]{4}{*}{$\mathrm{HF}(12 \mathrm{~W})$} & $\mathrm{WT}(n=5)$ & $805.3 \pm 39.7$ & $890.7 \pm 46.9$ & $403.1 \pm 49.3$ & $25.1 \pm 3.8$ \\
\hline & $\mathrm{ACl} \hat{0}$ & 316.6 & 224.5 & 221.4 & 37.6 \\
\hline & $\mathrm{AC} 2 \mathrm{O}$ & 436.1 & 297.1 & 226.0 & 40.3 \\
\hline & $\mathrm{AC} 3{ }^{\circ}$ & 399.0 & 308.3 & 239.5 & 38.5 \\
\hline
\end{tabular}




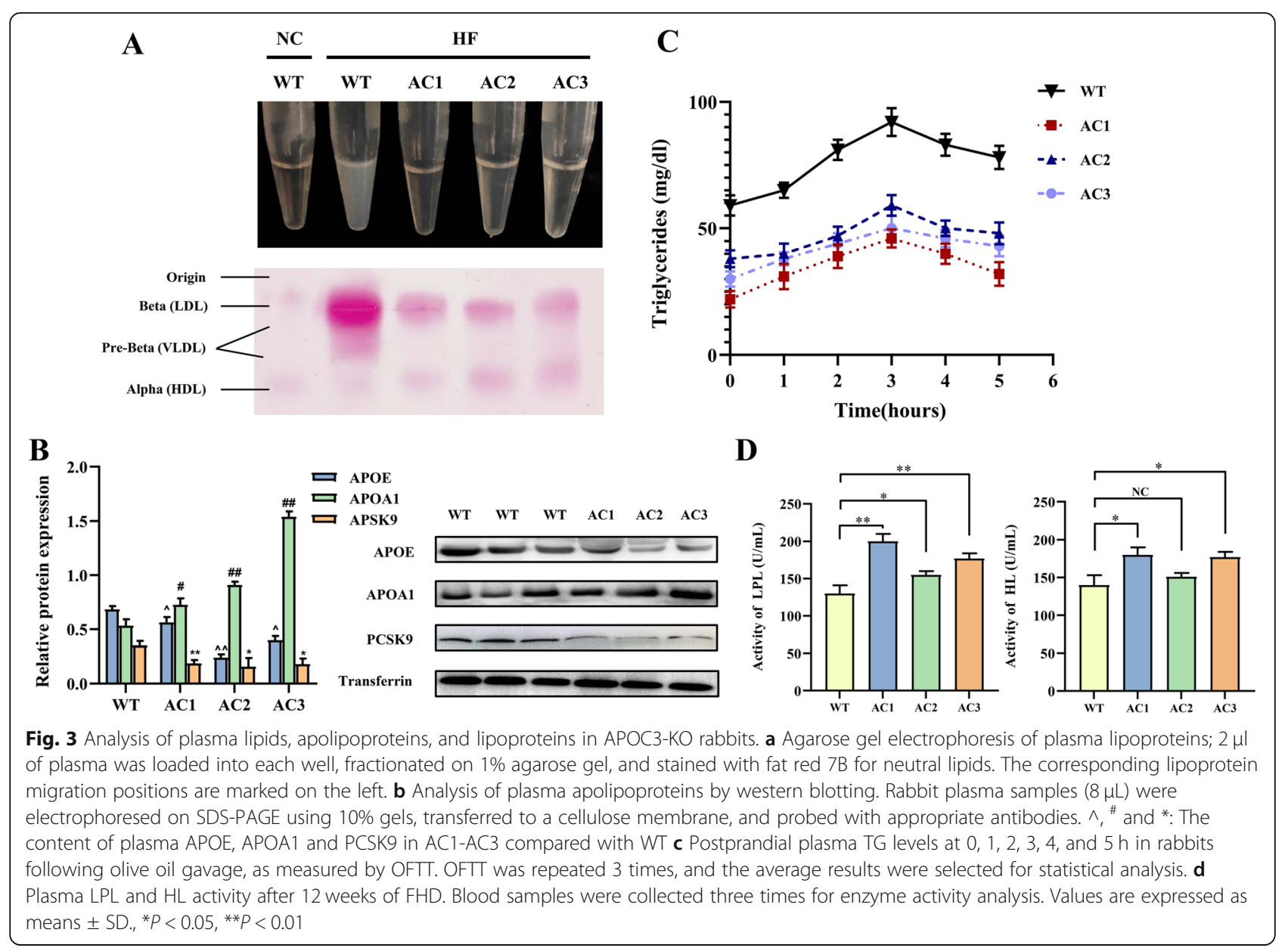

Analysis of atherosclerosis and liver pathological changes To directly observe the effects of APOC3 on the occurrence and development of atherosclerosis, we assessed the degree of atherosclerotic lesional severity in the coronary artery and aorta of the two groups of rabbits after the HFD regime. As shown in Fig. 4A, the WT rabbits had extensive plaque areas (stained bright red by the Sudan IV dye) in the aortic arch and smaller lesions scattered throughout the aorta. In contrast, only mild lesions were seen in the aorta of the APOC3-KO rabbits. The lesioned areas respectively comprised 21 and $3 \%$ of the aortic regions in the WT and APOC3-KO rabbits.

After 12 weeks of providing an HFD to WT rabbits, the HE, MT, anti-RAM-11, and $\alpha$-SMA staining of the beginning of the aorta showed hyperplastic intima and increased collagen contents accompanied by the accumulation of macrophages and proliferation of smooth muscle cells (Fig. 4B). In comparison, the aortic root lesions of the APOC3-KO rabbits were much milder. However, no significant changes were observed in the coronary arteries of either group (Fig. 4C), likely due to the short duration of the HFD. According to the results of the $\mathrm{HE}$ and Oil-red $\mathrm{O}$ staining, WT rabbits had significant accumulation of cytoplasmic lipid droplets in their livers, but less damage was observed in APOC3KO rabbits (Fig. 4D).

\section{Analysis of plasma inflammatory mediators and full blood count}

Atherosclerosis is a chronic inflammatory disease, and the inflammatory process is important in both the initiation and progression of lesion development. Therefore, we also analyzed levels of inflammatory cytokines (IL-1 $\beta$ and TNF- $\alpha$ ) and immune cell counts in the rabbits after 12 weeks of HFD. The APOC3-KO rabbits had fewer leukocytes and monocytes in their peripheral blood compared to the WT rabbits in addition to significantly lower serum inflammatory cytokine levels (Fig. 5A and B). Taken together, the evidence indicates APOC3 deficiency not only effectively reduced the number of macrophages in the atherosclerotic plaques but also had an effect on systemic inflammation.

\section{Discussion}

Hypertriglyceridemia is a sign of abnormal lipid metabolism and an independent risk factor in atherosclerotic 


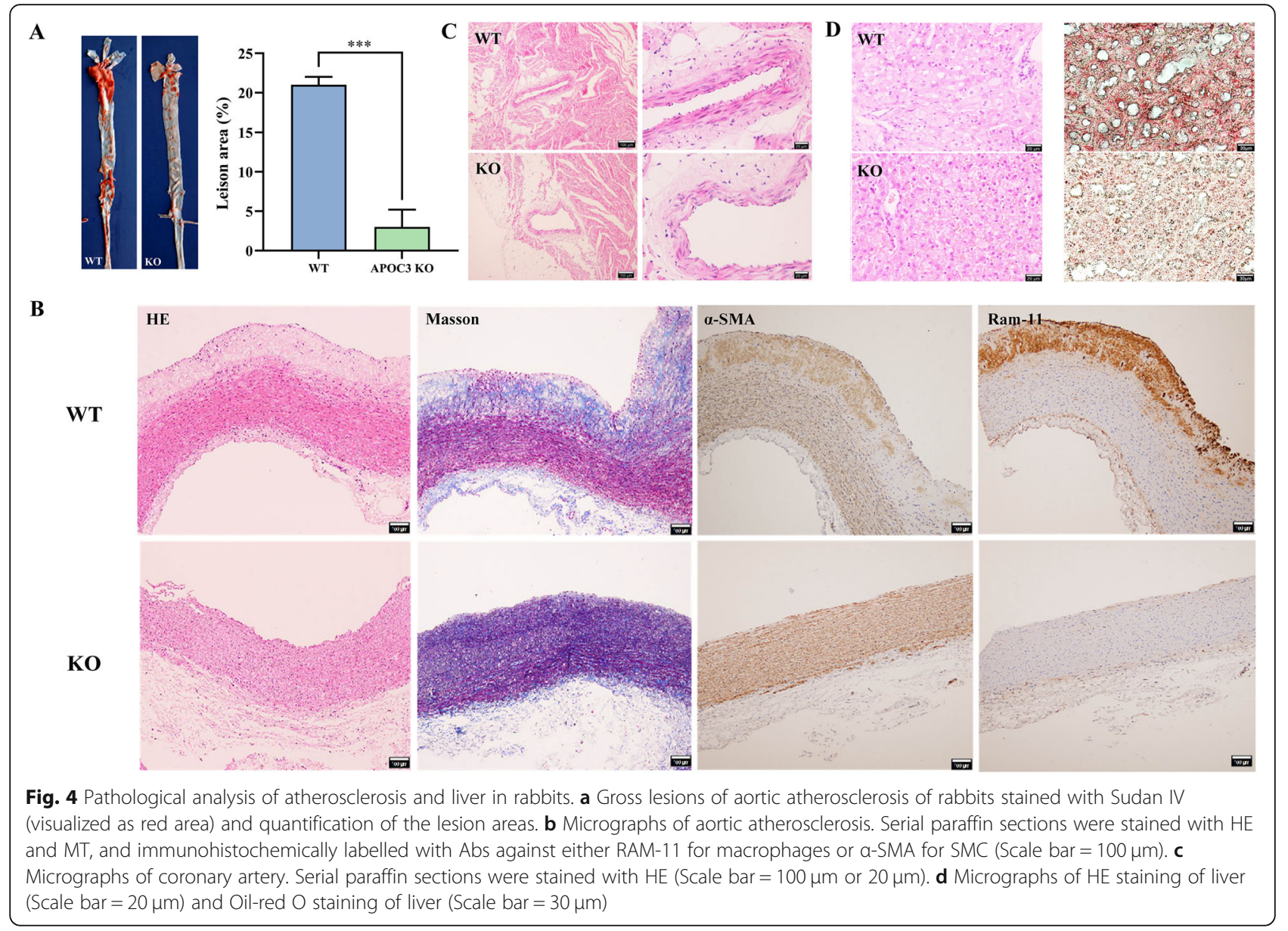

development [4]. APOC3, which is a key regulator of TG metabolism, is a water-soluble and low-molecular weight lipoprotein ubiquitous in the plasma along with HDLs, VLDLs, CM, and LDLs [22]. Studies show that elevated levels of APOC3 inhibit the activity of LPL and HL, which delays TG-rich lipoprotein clearance and increases TG levels in the plasma, eventually leading to impaired TG metabolism [23]. Although in vivo studies on APOC3 have mainly employed mouse models, rabbit models have several advantages, such as easier maintenance, suitable aortic dimensions, high fecundity, and short gestation periods [24], and they have similar lipid metabolism and cardiovascular pathophysiologies as humans [25]. For instance, the hepatic LDL receptor is normally inactive in rabbits, as in humans, which makes it a highly suitable model for studying the mechanistic basis of AS as well as the effects of lipid-lowering drugs [26]. Furthermore, rabbits have abundant plasma cholesteryl ester transfer protein, which can help when developing strategies to raise plasma HDL-C levels [27]. Finally, both humans and rabbits are more sensitive to an HFD than mice $[25,28]$.
APOC3 mainly comes from the liver, although the intestine is a secondary source, and it can be secreted into the blood [29]. We designed an sgRNA targeting exon 2 of rabbit APOC3 to finally obtain three $\mathrm{KO}$ rabbits by CRISPR/Cas9 gene-editing. None of the three founder rabbits contained APOC3 in their livers and small intestine, and no APOC3 protein was detected in their plasma. Under an NC diet, the plasma TG levels of the KO rabbits decreased, but the plasma TC and LDL-C levels were not significantly different compared with the control group. It is worth noting that after an HFD, in addition to plasma TG levels, plasma TC and LDL-C levels began to differ from the control group, indicating that a lack of APOC3 affects cholesterol transport, and this change is aggravated by a high-fat intake.

The plasma lipoprotein profiles of the WT and APOC3 KO rabbits showed significant differences; while APOB and APOE were significantly reduced in the APOC3 $\mathrm{KO}$ rabbits. APOA1 is the main apolipoprotein of plasma HDL [30], so the plasma APOA1 enrichment in $\mathrm{KO}$ rabbits leads to the increase of HDL level. This also confirmed that APOC3 may increase plasma TG levels by hindering APOE-mediated uptake of $\mathrm{CM}$ and 

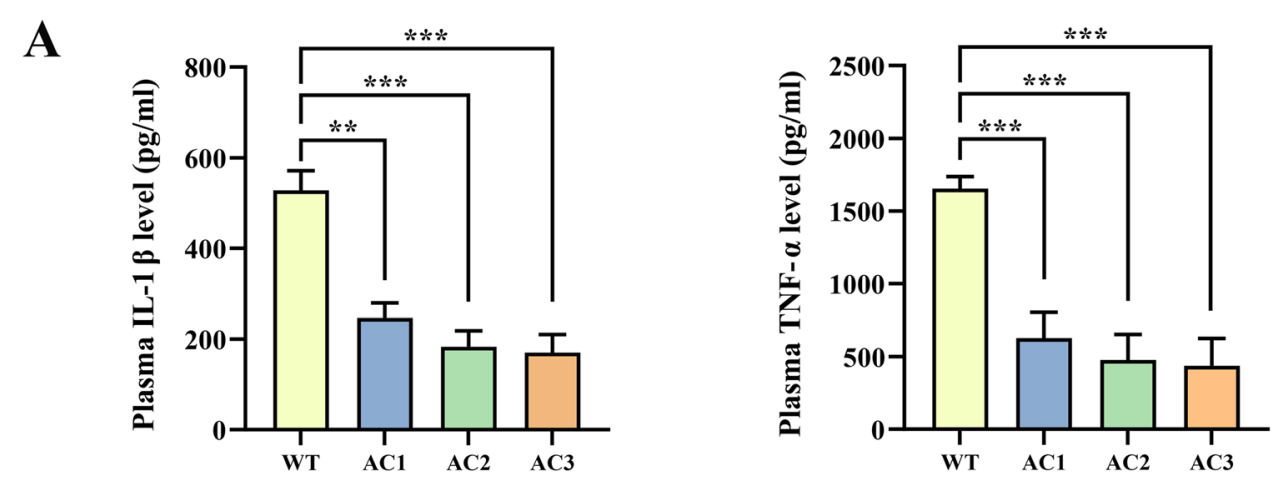

B
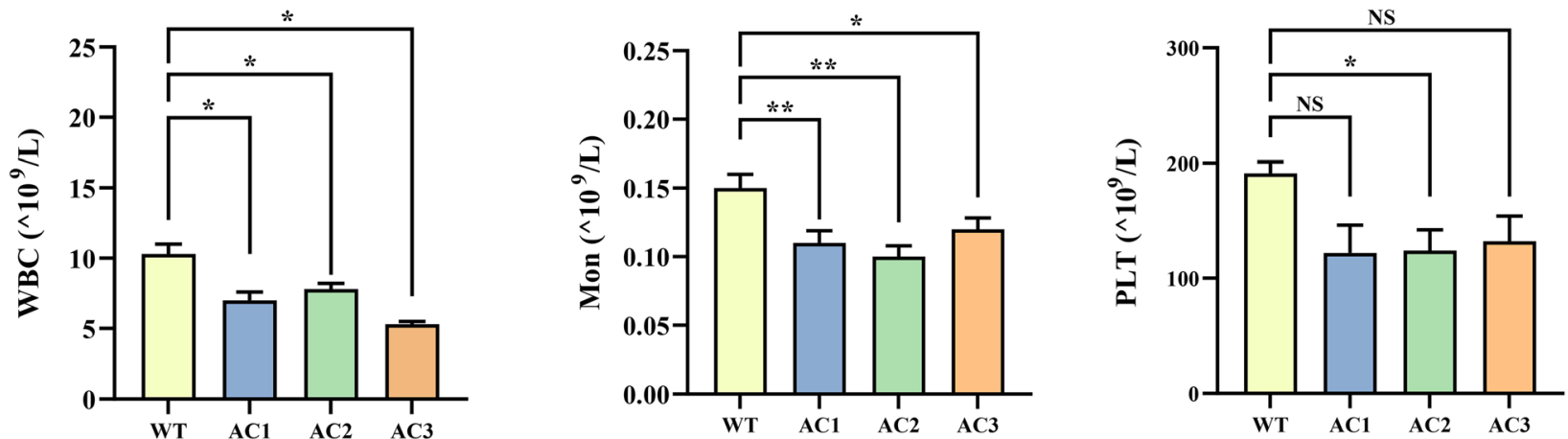

Fig. 5 Analysis of plasma inflammatory mediators and full blood count; blood samples were collected three times. Values are expressed as means $\pm S D$., ${ }^{*} P<0.05$, ${ }^{* *} P<0.01$, ${ }^{* * *} P<0.001$

VLDL in the liver by competitively inhibiting APOE binding to its receptor. We detected higher plasma LPL and $\mathrm{HL}$ activity in $\mathrm{AC} 1, \mathrm{AC} 2$ and $\mathrm{AC} 3$ than in WT rabbits, which is consistent with the results of most studies. However, in a previous study, APOC3 KO rabbits constructed using ZFN technology showed no difference in the activity of these two enzymes compared with the control group [31]. The sample size of our study was limited, and each rabbit had different genotypes, and each $\mathrm{KO}$ rabbit was separately divided into a group for statistical analysis. The inconsistent results of the two studies suggest that the correlation between APOC3 and LPL and HL activity needs to be further studied. At present, it is not clear whether APOC3 affects the expression of LPL.

Atherosclerosis is a chronic inflammatory disease, and the inflammatory process is important in both the initiation and progression of lesion development [32-34]. After detection, plasma IL- $1 \beta$ and TNF- $\alpha$ decreased significantly, and the whole-blood monocyte, neutrophil, and platelet counts were significantly lower in $\mathrm{KO}$ rabbits than WT rabbits. IL- $1 \beta$ and TNF- $\alpha$ can increase endothelial permeability and lipoprotein permeability $[35,36]$. Extensive monocyte recruitment plays an important role in the development of early atherosclerotic lesions [32, 37, 38], while neutrophils aggravate endothelial dysfunction, attract monocytes, enter atherosclerotic lesions, and accelerate the formation of foam cells [39]. Studies have confirmed that platelets contribute to the atherosclerotic process at both the early (endothelial disruption) and final stages (rupture of the vulnerable plaque), participating by releasing chemokines, inflammatory mediators, and microparticles [40, 41]. Therefore, it can be boldly speculated that APOC3 is closely related to the formation and development of atherosclerosis, in which a series of inflammatory responses play an important role.

After 12 weeks of HFD, the aortic tree lesion coverage of the WT rabbits was as high as $21 \%$, while that in KO rabbits was much lower (only $3 \%$ ). HE, MT, and immunohistochemical signals showed the WT rabbits had obvious atherosclerotic lesions, as well as increased intima thickening and collagen content, accompanied by the accumulation of macrophages and the proliferation of smooth muscle cells, while KO rabbits only had small early atherosclerotic lesions. However, there were no obvious pathological changes in the coronary arteries of the two groups. For the formation of coronary artery plaques in rabbits, higher blood lipids (such as seen in Watanabe heritable hyperlipidemic rabbits or LDLR KO rabbits) or other additional conditional interventions, such as hybridization or surgical ligation, are usually 
required. Taken together, the results suggest a lack of APOC3 restrains atherogenesis, although the exact mechanistic basis needs to be elucidated.

Statins are often used to treat dyslipidemia, especially in controlling LDL-C elevation; although clinicians generally believe that the benefits of statins are exaggerated, while the potential side effects are underestimated [42]. In addition, other risk factors for atherosclerosis still exist, such as TG and TG-rich lipoproteins [43]. However, the efficacy of existing TG-lowering drugs is still controversial. Therefore, using experimental animal models that are more similar to the characteristics of human lipid metabolism to study the associations between genes and cardiovascular disease may be conducive to the development and application of new drugs.

\section{Comparisons with other studies and what does the current work add to the existing knowledge}

APOC3 KO rabbits have previously been successfully constructed using the ZFN technique with adenine inserted into exon 3 of APOC3 gene [31]. In this study, APOC3-KO rabbits were achieved by anchoring exon 2 using the CRISPR/Cas9 gene editing technique that has becoming commonly used since its recent emergence. The two rabbit models were constructed in different ways. This indicates that the pathways for gene $\mathrm{KO}$ are diverse, and the gene target sites are selective.

\section{Study strengths and limitations}

For the first time, APOC3 KO rabbits were produced using the CRISPR/Cas9 system, and the models showed lower lipid levels and fewer atherosclerotic lesions after an HFD. However, only three APOC3 KO primary rabbits were obtained, and their genotypes were not completely consistent, even though, in the end, APOC3 was not detectable in the plasma, liver, or small intestine. The small sample size and different genotypes used were not conducive to the statistical analysis of the experimental data.

\section{Conclusion}

An APOC3-KO rabbit model was constructed using CRISPR/Cas9 gene editing technology. APOC3 deficiency alleviated cholesterol-induced hyperlipidemia and reduced atherosclerotic plaque formation. As the lipid metabolism profile of rabbits is similar to that of humans, this study provides an animal model for basic research on the anti-atherosclerotic effects of APOC3 inhibition therapy, in addition to providing information on the condition for drug development and clinical application.

\section{Abbreviations}

APOC3: Apolipoprotein C3; TG: Triglycerides; TC: Total cholesterol; LDL-

C: Low-density lipoprotein cholesterol; HDL-C: High-density lipoprotein cholesterol; VLDL-C: Very Low-density lipoprotein cholesterol; CM: Chylomicron; APOB: Apolipoprotein B; APOA1: Apolipoprotein A1; APOE: Apolipoprotein E; PCSK9: Proprotein convertase subtilisin/kexin type 9; LPL: Lipoprotein lipase; HL: Hepaticlipase; IL-1ß: Interleukin 1 beta; TNFa: Tumor Necrosis Factor-alpha; OFTT: Oral fat tolerance tests; HFD: High-fat diet; NC: Normal chow

\section{Supplementary Information}

The online version contains supplementary material available at https://doi. org/10.1186/s12944-021-01605-7.

Additional file 1: Supplement Fig. 1. Results of body weight of rabbits before and after HFD.

\section{Acknowledgments}

The authors would like to thank WOSCI(www.wosci.cn) for the English language review.

\section{Authors' contributions}

Jingyan Liang, Yong Cheng and Yingge Wang designed the experiments and provided the resources. Yiwen Zha and Yaoyao Lu performed the experiments, acquired and analyzed the data, and wrote the manuscript.

Ting Zhang, Kunning Yan and Wenwen Zhuang performed the experiments and acquired data. All authors have read and approved the final paper.

\section{Funding}

This work was supported by the National Key Research and Development Program of China, China (No. 2016YFE0126000).

Availability of data and materials

The datasets generated and/or analyzed during the current study are available from the corresponding author on request.

\section{Declarations}

Ethics approval and consent to participate

All protocols were approved by the Care and Use of Laboratory Animals (Ministry of Science and Technology of the People's Republic of China) and the Animal Care and Use Committee of Yangzhou University, Yangzhou, China (license number: SYXK (Su) 2017-0044).

Consent for publication

Not applicable.

\section{Competing interests}

The authors declare no conflicts of interest.

\section{Author details}

${ }^{1}$ Medical College, Yangzhou University, Yangzhou 225001, Jiangsu, China. ${ }^{2}$ College of Veterinary Medicine, Yangzhou University, Yangzhou 225009, Jiangsu, China. ${ }^{3}$ Institute of Translational Medicine, Medical College, Yangzhou University, Yangzhou 225001, Jiangsu, China. ${ }^{4}$ Jiangsu Key Laboratory of Integrated Traditional Chinese and Western Medicine for Prevention and Treatment of Senile Diseases, Yangzhou University, Yangzhou 225001, China. ${ }^{5}$ Jiangsu Co-Innovation Center for Prevention and Control of Important Animal Infectious Disease and Zoonoses, Yangzhou 225001, China. ${ }^{6}$ Affiliated Hospital of Yangzhou University, Yangzhou 225001, Jiangsu, China.

Received: 13 September 2021 Accepted: 26 November 2021

Published online: 18 December 2021

\section{References}

1. Masucci-Magoulas L, Goldberg IJ, Bisgaier CL, Serajuddin H, Francone OL, Breslow $J$, et al. A mouse model with features of familial combined hyperlipidemia. Science. 1997;275(5298):391-4. https://www.ncbi.nlm.nih. gov/pubmed/8994037. https://doi.org/10.1126/science.275.5298.391.

2. Timpson NJ, Walter $\mathrm{K}, \mathrm{Min} J \mathrm{~L}$, et al. A rare variant in APOC3 is associated with plasma triglyceride and VLDL levels in Europeans. Nat Commun. 2014; 5:4871 https://www.ncbi.nlm.nih.gov/pubmed/25225788. 
3. Lawler PR, Kotrri G, Koh M, Goodman SG, Farkouh ME, Lee DS, et al. Real-world risk of cardiovascular outcomes associated with hypertriglyceridaemia among individuals with atherosclerotic cardiovascular disease and potential eligibility for emerging therapies. Eur Heart J. 2020;41(1):86-94. https://www.ncbi.nlm. nih.gov/pubmed/31733058. https://doi.org/10.1093/eurheartj/ehz767.

4. Reiner Z. Hypertriglyceridaemia and risk of coronary artery disease. Nat Rev Cardiol. 2017;14(7):401-11. https://www.ncbi.nlm.nih.gov/pubmed/283 00080. https://doi.org/10.1038/nrcardio.2017.31.

5. $\mathrm{Tg} \mathrm{NHL}, \mathrm{Hdl}$ Working Group of the Exome Sequencing Project, I. Blood, et al. Loss-of-function mutations in APOC3, triglycerides, and coronary disease. N Engl J Med. 2014;371:22-31 https://www.ncbi.nlm.nih.gov/ pubmed/24941081.

6. Li H, Han Y, Qi R, Wang Y, Zhang X, Yu M, et al. Aggravated restenosis and atherogenesis in ApoCIII transgenic mice but lack of protection in ApoCIII knockouts: the effect of authentic triglyceride-rich lipoproteins with and without ApoCIII. Cardiovasc Res. 2015;107(4):579-89. https://www.ncbi.nlm. nih.gov/pubmed/26160324. https://doi.org/10.1093/cvr/cvv192.

7. Fan J, Chen Y, Yan H, Niimi M, Wang Y, Liang J. Principles and applications of rabbit models for atherosclerosis research. J Atheroscler Thromb. 2018; 25(3):213-20. https://www.ncbi.nlm.nih.gov/pubmed/29046488. https://doi. org/10.5551/jat.RV17018.

8. Wang W, Chen Y, Bai L, et al. Transcriptomic analysis of the liver of cholesterol-fed rabbits reveals altered hepatic lipid metabolism and inflammatory response. Sci Rep. 2018;8:6437 https://www.ncbi.nlm.nih.gov/ pubmed/29692426.

9. Pankowicz FP, Barzi M, Kim KH, et al. Rapid Disruption of Genes Specifically in Livers of Mice Using Multiplex CRISPR/Cas9 Editing. Gastroenterology. 2018;155:1967-70 https://www.ncbi.nlm.nih.gov/pubmed/30170115.

10. Lu R, Yuan T, Wang Y, Zhang T, Yuan Y, Wu D, et al. Spontaneous severe hypercholesterolemia and atherosclerosis lesions in rabbits with deficiency of low-density lipoprotein receptor (LDLR) on exon 7. EBioMedicine. 2018; 36:29-38. https://www.ncbi.nlm.nih.gov/pubmed/30243490. https://doi. org/10.1016/j.ebiom.2018.09.020.

11. Yuan T, Zhong Y, Wang Y, et al. Generation of hyperlipidemic rabbit models using multiple sgRNAs targeted CRISPR/Cas9 gene editing system. Lipids Health Dis. 2019;18:69 https://www.ncbi.nlm.nih.gov/pubmed/30885208.

12. Zou Q, Wang X, Liu Y, Ouyang Z, Long H, Wei S, et al. Generation of genetarget dogs using CRISPR/Cas9 system. J Mol Cell Biol. 2015;7(6):580-3. https://www.ncbi.nlm.nih.gov/pubmed/26459633. https://doi.org/10.1093/ jmcb/mjv061.

13. Guo M, Xu Y, Dong Z, Zhou Z, Cong N, Gao M, et al. Inactivation of ApoC3 by CRISPR/Cas9 protects against atherosclerosis in hamsters. Circ Res. 2020; 127(11):1456-8. https://www.ncbi.nlm.nih.gov/pubmed/32951534. https:// doi.org/10.1161/CIRCRESAHA.120.317686.

14. Kim H, Kim JS. A guide to genome engineering with programmable nucleases. Nat Rev Genet. 2014;15(5):321-34. https://www.ncbi.nlm.nih.gov/ pubmed/24690881. https://doi.org/10.1038/nrg3686.

15. Karimian A, Azizian K, Parsian H, Rafieian S, Shafiei-Irannejad V, Kheyrollah M, et al. CRISPR/Cas9 technology as a potent molecular tool for gene therapy. J Cell Physiol. 2019;234(8):12267-77. https://www.ncbi.nlm.nih.gov/ pubmed/30697727. https://doi.org/10.1002/jcp.27972

16. Chadwick AC, Musunuru K. Genome Editing for the Study of Cardiovascular Diseases. Curr Cardiol Rep. 2017;19:22 https://www.ncbi.nlm.nih.gov/ pubmed/28220462.

17. Niimi M, Yang D, Kitajima S, Ning B, Wang C, Li S, et al. ApoE knockout rabbits: a novel model for the study of human hyperlipidemia. Atherosclerosis. 2016;245:187-93. https://www.ncbi.nlm.nih.gov/pubmed/2 6724529. https://doi.org/10.1016/j.atherosclerosis.2015.12.002.

18. Shiomi M, Ito T, Yamada S, Kawashima S, Fan J. Development of an animal model for spontaneous myocardial infarction (WHHLMI rabbit). Arterioscler Thromb Vasc Biol. 2003;23(7):1239-44. https://www.ncbi.nlm.nih.gov/ pubmed/12738683. https://doi.org/10.1161/01.ATV.0000075947.28567.50.

19. Pirillo A, Catapano AL. Mutations of APOC3 gene, metabolism of triglycerides and reduction of ischemic cardiovascular events. G Ital Cardio (Rome). 2015;16:289-94 https://www.ncbi.nlm.nih.gov/pubmed/25994465.

20. Xu QY, Li H, Cao HX, et al. APOC3 rs2070667 Associates with Serum Triglyceride Profile and Hepatic Inflammation in Nonalcoholic Fatty Liver Disease. Biomed Res Int. 2020;2020:8869674 https://www.ncbi.nlm.nih.gov/ pubmed/33294458.

21. Khetarpal SA, Zeng X, Millar JS, Vitali C, Somasundara AVH, Zanoni P, et al. A human APOC3 missense variant and monoclonal antibody accelerate apoC-
III clearance and lower triglyceride-rich lipoprotein levels. Nat Med. 2017; 23(9):1086-94. https://www.ncbi.nlm.nih.gov/pubmed/28825717. https://doi. org/10.1038/nm.4390.

22. Khetarpal SA, Qamar A, Millar JS, et al. Targeting ApoC-III to Reduce Coronary Disease Risk. Curr Atheroscler Rep. 2016;18:54 https://www.ncbi. nlm.nih.gov/pubmed/27443326.

23. Norata GD, Tsimikas S, Pirillo A, Catapano AL. Apolipoprotein C-III: from pathophysiology to pharmacology. Trends Pharmacol Sci. 2015;36(10):67587. https://www.ncbi.nlm.nih.gov/pubmed/26435212. https://doi.org/10.101 6/j.tips.2015.07.001.

24. Getz GS, Reardon CA. Animal models of atherosclerosis. Arterioscler Thromb Vasc Biol. 2012;32(5):1104-15. https://www.ncbi.nlm.nih.gov/pubmed/22383 700. https://doi.org/10.1161/ATVBAHA.111.237693.

25. Fan J, Watanabe T. Cholesterol-fed and transgenic rabbit models for the study of atherosclerosis. J Atheroscler Thromb. 2000;7(1):26-32. https:// www.ncbi.nlm.nih.gov/pubmed/11425041. https://doi.org/10.5551/jat1994. 7.26 .

26. Fan J, Kitajima S, Watanabe T, Xu J, Zhang J, Liu E, et al. Rabbit models for the study of human atherosclerosis: from pathophysiological mechanisms to translational medicine. Pharmacol Ther. 2015;146:104-19. https://www. ncbi.nlm.nih.gov/pubmed/25277507. https://doi.org/10.1016/j.pharmthera.2 014.09.009.

27. Zhang J, Niimi M, Yang D, Liang J, Xu J, Kimura T, et al. Deficiency of cholesteryl Ester transfer protein protects against atherosclerosis in rabbits. Arterioscler Thromb Vasc Biol. 2017;37(6):1068-75. https:/www.ncbi.nlm.nih. gov/pubmed/28428219. https://doi.org/10.1161/ATVBAHA.117.309114.

28. Taylor JM, Fan J. Transgenic rabbit models for the study of atherosclerosis. Front Biosci. 1997;2(4):d298-308. https://www.ncbi.nlm.nih.gov/pubmed/92 06988. https://doi.org/10.2741/A192.

29. Ramms B, Gordts P. Apolipoprotein C-III in triglyceride-rich lipoprotein metabolism. Curr Opin Lipidol. 2018;29(3):171-9. https://www.ncbi.nIm.nih. gov/pubmed/29547399. https://doi.org/10.1097/MOL.0000000000000502.

30. Cochran BJ, Ong KL, Manandhar B, et al. APOA1: a Protein with Multiple Therapeutic Functions. Curr Atheroscler Rep. 2021;23:11 https://www.ncbi. nlm.nih.gov/pubmed/33591433.

31. Yan H, Niimi M, Matsuhisa F, Zhou H, Kitajima S, Chen Y, et al. Apolipoprotein CIII deficiency protects against atherosclerosis in knockout rabbits. Arterioscler Thromb Vasc Biol. 2020;40(9):2095-107. https://www. ncbi.nlm.nih.gov/pubmed/32757647. https://doi.org/10.1161/ATVBAHA.12 0.314368 .

32. Tunon J, Badimon L, Bochaton-Piallat ML, et al. Identifying the antiinflammatory response to lipid lowering therapy: a position paper from the working group on atherosclerosis and vascular biology of the European Society of Cardiology. Cardiovasc Res. 2019;115(1):10-9. https://www.ncbi. nlm.nih.gov/pubmed/30534957. https://doi.org/10.1093/cvr/cvy293.

33. Tousoulis D, Oikonomou E, Economou EK, Crea F, Kaski JC. Inflammatory cytokines in atherosclerosis: current therapeutic approaches. Eur Heart J. 2016;37(22):1723-32. https://www.ncbi.nlm.nih.gov/pubmed/26843277. https://doi.org/10.1093/eurheartj/ehv759.

34. Valenzuela CA, Baker EJ, Miles EA, et al. Eighteencarbon trans fatty acids and inflammation in the context of atherosclerosis. Prog Lipid Res. 2019;76: 101009 https://www.ncbi.nlm.nih.gov/pubmed/31669459.

35. Oorni K, Lehti S, Sjovall P, et al. Triglyceride-rich lipoproteins as a source of Proinflammatory lipids in the Arterial Wall. Curr Med Chem. 2019;26(9): 1701-10. https://www.ncbi.n/m.nih.gov/pubmed/29848270. https://doi.org/1 0.2174/0929867325666180530094819.

36. Grebe A, Hoss F, Latz E. NLRP3 Inflammasome and the IL-1 pathway in atherosclerosis. Circ Res. 2018;122(12):1722-40. https://www.ncbi.nlm.nih. gov/pubmed/29880500. https://doi.org/10.1161/CIRCRESAHA.118.311362.

37. Tabas I, Lichtman AH. Monocyte-macrophages and T cells in atherosclerosis. Immunity. 2017:47(4):621-34. https://www.ncbi.nlm.nih.gov/pubmed/2904 5897. https://doi.org/10.1016/j.immuni.2017.09.008.

38. Quintar A, McArdle S, Wolf D, Marki A, Ehinger E, Vassallo M, et al. Endothelial protective monocyte patrolling in large arteries intensified by Western diet and atherosclerosis. Circ Res. 2017;120(11):1789-99. https:// www.ncbi.nlm.nih.gov/pubmed/28302649. https://doi.org/10.1161/CIRCRESA HA.117.310739.

39. Doring $Y$, Soehnlein $O$, Weber $C$. Neutrophil extracellular traps in atherosclerosis and Atherothrombosis. Circ Res. 2017;120(4):736-43. https:// www.ncbi.nlm.nih.gov/pubmed/28209798. https://doi.org/10.1161/CIRCRESA HA.116.309692. 
40. Papapanagiotou A, Daskalakis G, Siasos G, Gargalionis A, G. Papavassiliou A. The role of platelets in cardiovascular disease: molecular mechanisms. Curr Pharm Des. 2016;22(29):4493-505. https://www.ncbi.n/m.nih.gov/pubmed/2 7281334. https://doi.org/10.2174/1381612822666160607064118.

41. Barrett TJ, Schlegel M, Zhou F, et al. Platelet regulation of myeloid suppressor of cytokine signaling 3 accelerates atherosclerosis. Sci Transl Med. 2019;11 https://www.ncbi.nlm.nih.gov/pubmed/31694925.

42. Mahase E. MP calls for independent review of benefits and harms of statins. BMJ. 2019;366:15380 https://www.ncbi.nlm.nih.gov/pubmed/31481358.

43. Vallejo-Vaz AJ, Corral P, Schreier L, Ray KK. Triglycerides and residual risk. Curr Opin Endocrinol Diabetes Obes. 2020;27(2):95-103. https://www.ncbi. nlm.nih.gov/pubmed/32073428. https://doi.org/10.1097/MED. 0000000000000530

\section{Publisher's Note}

Springer Nature remains neutral with regard to jurisdictional claims in published maps and institutional affiliations.

Ready to submit your research? Choose BMC and benefit from:

- fast, convenient online submission

- thorough peer review by experienced researchers in your field

- rapid publication on acceptance

- support for research data, including large and complex data types

- gold Open Access which fosters wider collaboration and increased citations

- maximum visibility for your research: over $100 \mathrm{M}$ website views per year

At $\mathrm{BMC}$, research is always in progress.

Learn more biomedcentral.com/submissions 